\title{
ㄷำ1
}

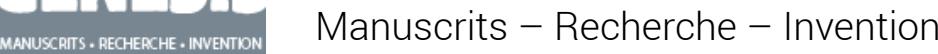

$50 \mid 2020$

Aragon

\section{Le bon génie de la genèse}

\section{Pierre-Marc de Biasi}

\section{OpenEdition}

\section{Journals}

Édition électronique

URL : https://journals.openedition.org/genesis/5148

DOI : 10.4000/genesis. 5148

ISSN : 2268-1590

\section{Éditeur :}

Presses universitaires de Paris Sorbonne (PUPS), Société internationale de génétique artistique littéraire et scientifique (SIGALES)

\section{Édition imprimée}

Date de publication : 15 juillet 2020

Pagination : 19-32

ISBN : 979-10-231-0679-4

ISSN : $1167-5101$

\section{Référence électronique}

Pierre-Marc de Biasi, « Le bon génie de la genèse », Genesis [En ligne], 50 | 2020, mis en ligne le 15 juillet 2021, consulté le 12 janvier 2023. URL : http://journals.openedition.org/genesis/5148 ; DOI https://doi.org/10.4000/genesis.5148 


\title{
Le bon génie de la genèse
}

\author{
Pierre-Marc de Biasi
}

$\mathrm{D}$

ifficile d'évoquer ici la figure d'Aragon sans rappeler le rôle décisif qu'il a joué, en personne, dans l'édification de la «génétique littéraire» comme nouvelle discipline critique. Homme d'appareil mais visionnaire et imprévisible, ironique mais passionné de création et d'idées nouvelles, Louis Aragon s'est laissé convaincre, au cours de ses dernières années, par les projets d'un petit groupe d'écrivains, d'universitaires et de chercheurs qui s'étaient mis en tête de révolutionner le regard critique en donnant une place centrale aux archives littéraires autour de la question : les brouillons, les notes et les documents de travail des créateurs, foisonnants dans les bibliothèques mais délaissés par les critiques, ne seraient-ils pas une voie royale pour renouveler notre connaissance des œuvres en nous permettant d'entrer dans le laboratoire de l'écrivain? Tout en réfléchissant sur le sort institutionnel qu'il allait lui-même réserver à ses propres manuscrits, Louis Aragon s'est intéressé de très près à ces «études de genèse » entreprises, à l'initiative de Louis Hay, sur quelques grands corpus (Heine, Proust, Valéry, Flaubert, Zola, etc.), par une poignée de jeunes équipes qui s'occupaient, à l'École normale supérieure, de bâtir un socle théorique à ce type de recherche. Et il s'y est intéressé de si près qu'il aura finalement été celui par qui l'approche génétique, à l'état naissant, a pu passer du statut de prototype expérimental à celui de dispositif opérationnel.

Faisant du legs de ses propres archives, en 1977, l'enjeu d'une véritable négociation sur le traitement scientifique des manuscrits modernes, Louis Aragon aura pesé, du poids considérable de toute son œuvre, pour convaincre l'État de transformer, cinq ans plus tard, la petite structure provisoire créée par Louis Hay - le CAM (Centre d'analyse des manuscrits) en une unité pérenne de recherche, l'ITEM (Institut des textes et manuscrits modernes) : un «laboratoire propre» du CNRS, qui allait devenir, jusqu' aujourd'hui, le pôle initiateur et fédérateur de toutes les investigations menées sur la genèse des œuvres, en France comme à l'étranger. C'est sur le sens de cette initiative que je voudrais revenir non seulement pour souligner le rôle qu'Aragon a effectivement joué dans la reconnaissance de notre discipline, mais aussi pour essayer de comprendre dans quelle logique symbolique son engagement a pu s'inscrire, et par quelle affinité élective (mais aussi avec quel décalage ironique) son point de vue personnel sur la création a pu si spontanément se trouver en accord avec les principes de cette nouvelle méthodologie critique.

L'étude de genèse est fondamentalement indicielle. Qu'il s'agisse de textes ou de toute autre forme de création intellectuelle, artistique ou scientifique, l'approche génétique des œuvres dépend de la quantité et de la qualité des traces qui, si elles sont assez complètes, permettent aux chercheurs de reconstituer et de comprendre les processus qui ont donné naissance et forme à l'œuvre définitive. Il est très rare que l'existence même de ces traces

Le patrimoine des écrivains en héritage 
soit le fruit du hasard. Le fait que des archives de travail (notes, brouillons, plans, esquisses, croquis, ébauches, documents, etc.) aient pu être conservées suppose qu'elles aient d'abord été l'objet d'une attention personnelle et persistante du créateur lui-même qui, au lieu de s'en désintéresser, de les laisser se perdre ou de s'en débarrasser une fois son travail terminé, a pris soin au fil des années de les garder, de les classer et de les protéger pendant toute sa vie, puis de les réunir et de les transmettre, après sa mort, comme un témoignage de son projet, de ses idées, de ses techniques et de sa démarche.

À ce premier miracle intentionnel et durable qui tient à la croyance du créateur en ses archives et au souci qu'il a entretenu tout au long de son existence de pourvoir à leur sauvegarde et à leur transmission, s'ajoute un second miracle, qui cette fois ne dépend même plus de lui : la bonne fortune d'une issue heureuse aux péripéties des héritages, des conflits familiaux, des partages inopportuns, des dégâts des eaux, incendies et autres aléas historiques qui président au destin mouvementé des archives après la disparition de leur auteur. Il faut une accumulation prodigieuse de hasards bienveillants pour que ces documents fragiles qui transitent entre différentes mains (héritiers, ayants droit, amis, collectionneurs, marchands, etc.) parfois pendant plusieurs générations, échappent aux dangers et aux convoitises, et finissent par rejoindre un statut pérenne et un espace sécurisé où ils pourront traverser le temps sans risque d'être détruits, perdus, éparpillés ou détournés, accaparés, séquestrés et finalement rendus inaccessibles.

\section{De la transmission à la redécouverte}

La bibliothèque publique et le musée sont les institutions d'État qui ont été inventées pour répondre à cette exigence, mais ce sont des institutions récentes qui, jusqu'à la fin du XIXe siècle, n'avaient pas vocation à conserver autre chose que des textes publiés ou des œuvres abouties, et nullement des dossiers de genèse faits de brouillons raturés, d'ébauches approximatives ou de dessins préparatoires. Il a fallu redéfinir l'instrument institutionnel pour qu'il élargisse ses compétences à la sauvegarde et la valorisation de ces archives, et cette redéfinition, c'est bel et bien à l'action personnelle des écrivains et des artistes que nous la devons. Ce ne sont pas les conservateurs mais les créateurs qui ont opéré et légitimé cette conversion du regard par laquelle le document de genèse s'est trouvé élevé au statut d'objet précieux et significatif, digne d'être sauvegardé et valorisé. Que les créateurs aient toujours eu plus d'une longueur d'avance sur les institutions culturelles chargées de veiller à la survie de leurs œuvres, c'est ce que l'histoire nous démontre à l'envi. Dans le monde occidental qui invente les Lumières, le Progrès, l'idée de génie, le concept de propriété intellectuelle, le droit d'auteur, la valeur du mérite et une idée nouvelle du travail, les écrivains de la seconde moitié du XviII siècle (Diderot, Rousseau, Schiller, etc.) avaient pris conscience de l'intérêt qu'il pouvait y avoir, pour l'avenir, à conserver leurs documents de travail. Les écrivains du XIXe siècle (Goethe, Hugo, Poe, Flaubert, etc.) en ont théorisé le principe épistémologique en termes de théorie de la création, puis en ont construit les conditions de possibilité technique en imposant que soient créées les structures permettant la conservation de ce nouveau patrimoine. En France, c'est Victor Hugo qui a joué le rôle déterminant d'éveilleur de consciences et de maître d'œuvre, en programmant lui-même le protocole et le lieu de sauvegarde de ses manuscrits.

Mais il a fallu attendre encore près d'un siècle pour que ces archives, désormais disponibles à la recherche mais traitées de manière éclectique par l'histoire littéraire, suscitent la 
méthodologie et les concepts critiques capables de les élucider et de les comprendre comme traces de la genèse de l'œuvre, c'est-à-dire comme indices permettant de reconstituer et d'interpréter l'œuvre en termes de processus. Pour des raisons épistémologiques et sociologiques d'ailleurs passionnantes à suivre dans leur développement, les conditions intellectuelles d'une telle réévaluation des manuscrits n'ont pas pu être réunies avant le «moment théorique » des années 1960-1970 qui s'est caractérisé par l'irruption des sciences de l'homme dans le champ critique, la remise en cause de l'auteur et la mise à l'index du manuscrit d'écrivain au profit d'un intérêt presque exclusif pour la structure formelle des significations et la clôture du texte sur lui-même. Mais c'est ce «nettoyage par le vide» qui a finalement permis de faire émerger une image critique du manuscrit radicalement inédite : au lieu de le considérer comme une pauvre chose biographique, abusivement fétichisée par l'histoire littéraire, ou comme un inépuisable réservoir de «sources », à la discrétion d'une critique positiviste en mal de preuves, on a pu commencer à y voir l'espace même dans lequel s'était concrètement joué et exercé le fameux «travail du texte» que le moment théorique avait voulu faire prévaloir comme dynamique même du sens... À partir de cet instant, la «génétique » était née, dans le sillage du structuralisme, comme la promesse d'une nouvelle épistémè critique : il devenait possible de la comprendre comme une hypothèse méthodologique entièrement neuve, mais qui aurait été préparée et comme préméditée pendant deux siècles par des générations d'écrivains. Car il est vite apparu que cette nouvelle discipline se trouvait de fait «sous influence» et redevable d'un double parrainage : en amont, la contribution essentielle des créateurs qui, comme Hugo, avaient organisé, depuis la fin du XVIII e siècle, la sauvegarde des matériaux - les documents de genèse - qui constituent l'objet même de la critique génétique; et de manière contemporaine, au moment même où cette approche critique prenait son essor en tant que telle, le soutien des créateurs qui, comme Aragon (mais aussi Ponge, Sartre, Butor, etc.), permettaient à la discipline de faire reconnaître sa légitimité et aidaient les chercheurs à se doter des structures indispensables à leurs travaux.

En termes de structure de sauvegarde, la critique génétique, née dans les années 1970, peut donc être considérée comme le lointain effet scientifique d'un texte fondateur qui a conduit la Bibliothèque nationale de France à ouvrir à la production contemporaine et aux manuscrits de travail un département des Manuscrits occidentaux où sont venus s'accumuler, tout au long du $\mathrm{xx}^{\mathrm{e}}$ siècle, les fonds autographes de documents de genèse qui se comptent aujourd'hui par millions de pièces, encore largement inédits. Chacun sait que ce texte fondateur - le Codicille de 1881 - est le testament par lequel Victor Hugo, quatre ans avant d'être inhumé au Panthéon, léguait la totalité de ses manuscrits à la Nation en donnant à ce corpus, promis à la survie éternelle, le sens métaphorique d'un corps glorieux qui serait le double durable de sa personne, et la valeur symbolique d'un « œil spirituel» exigeant et vigilant, perpétuellement ouvert sur l'avenir. J'ai longuement commenté ce Codicille, ses termes, ses raisons, ses attendus et ses conséquences, dans un numéro précédent de la revue Genesis consacré à Hugo ${ }^{1}$, et je n'y reviens pas ici. Mais les deux questions sont indissociables : pour

\section{Le cycle long des gestes symboliques}

1. Pierre-Marc de Biasi, «"Je donne tous mes manuscrits..." ou Les deux corps de l'écrivain», Genesis, n 45, «Hugo», SUP, 2017, p. 32-46. 
comprendre le geste d'Aragon, il faut vraiment en revenir à Hugo. Non seulement Aragon connaissait parfaitement le Codicille, mais son exemplarité s'est visiblement imposée à lui tout au long de sa réflexion sur la postérité de ses propres archives.

Ce que veut dire le legs de 1881 est tout entier présent dans le legs de 1977, et il va de soi que pour Aragon, ce qui se joue dans ses propres dispositions testamentaires a le sens d'un véritable rendez-vous personnel devant l'Histoire avec celui qu'il considère comme la figure tutélaire la plus haute de la littérature française : l'écrivain total auquel il n'a cessé de penser et de se mesurer, dont il s'est inspiré, qu'il a commenté et admiré... celui dont il voudrait avoir été le retour, la continuation ou l'équivalent au $x^{2}$ siècle. C'est donc évidemment le contraire d'un hasard si, un siècle exactement après Victor Hugo, dans un geste testamentaire et solennel qui redouble celui du Codicille, Louis Aragon, six ans avant de disparaître, décide à son tour de léguer la totalité de ses manuscrits à la Nation. Il s'inscrit résolument dans la même logique, tout en décalant le modèle hugolien : en cherchant à provoquer, dans notre relation à la mémoire des écrivains, une mutation culturelle aussi irréversible que celle initiée par Hugo, mais en la portant à une nouvelle échelle et en la déportant dans un nouveau registre. Hugo avait légitimé le manuscrit de travail, le brouillon, la rature comme biens symboliques dignes de conservation et de consécration; Aragon surenchérit mais marque aussi son propre terrain en les légitimant non comme trésors patrimoniaux dignes de vénération mais comme objets scientifiques appelant un dispositif de recherche et d'analyse critique.

Et voilà pourquoi, au lieu de léguer ses manuscrits à la $\mathrm{BnF}$, comme chaque écrivain le faisait depuis Victor Hugo, Aragon décide de les donner expressément au CNRS qu'il désigne par ce geste comme le nouvel espace où, au-delà de la sauvegarde et de la postérité institutionnelle, les documents de genèse des œuvres pourront rencontrer, au Xxie siècle, le véritable destin qu'il leur assigne : celui de reprendre vie et actualité, en tant qu'objets-sujets d'investigation, grâce au travail et à l'empathie des chercheurs. Ce legs était articulé à un dispositif comprenant, comme chez Hugo, des dispositions précises touchant les personnes qui seraient chargées de valoriser les fonds, d'en dresser l'inventaire et de mener les premières opérations de recherche : des proches, assez jeunes pour assurer l'avenir du projet, à commencer par Jean Ristat, compagnon des dernières années et légataire universel, et Michel Apel-Muller, ami d'Aragon et spécialiste d'Elsa Triolet, puisque le legs regroupait les deux corpus en un seul fonds, indissociable comme le couple mythique qu'avaient formé Louis et Elsa.

\section{Le cycle court des circonstances}

Déclaration solennelle de legs en juin 1976, dépôt des manuscrits et discours inaugural devant le directeur du CNRS en mai 1977 : incertain sur sa propre longévité, Aragon, déjà octogénaire, a préféré ne pas attendre 1981 - le centième anniversaire du Codicille - pour procéder au legs effectif de ses manuscrits. Le CNRS ne s'est pas fait prier pour accepter le cadeau, mais, moins inquiet sur ses propres échéances, toujours circonspect devant les nouvelles dépenses et un peu embarrassé par la question technique (comment conserver près de 80000 documents originaux ?), le ministère s'est accordé encore quelques années de réflexion avant de créer le grand laboratoire, promis en échange, qui devait accueillir les fonds et la nouvelle équipe Aragon-Triolet. Ce sera pourtant chose faite quatre ans plus tard, en 1982, avec la fondation officielle de l'ITEM, quelques mois avant la disparition de l'écrivain le 24 décembre : Aragon aura donc eu la satisfaction de voir son projet intégralement 
réalisé de son vivant. Il faut dire qu'entre-temps bien des choses avaient changé dans le pays et que le CNRS n'avait peut-être plus d'autre choix que d'obtempérer : l'année 1981 n'avait pas seulement marqué le centenaire du Codicille, elle avait aussi vu la victoire électorale de l'union de la gauche, l'arrivée de Mitterrand à l'Élysée et le retour de ministres communistes au gouvernement... Dans ces circonstances, comment tarder plus longtemps pour honorer la promesse faite à celui que l'on considérait comme le plus grand écrivain communiste français du $\mathrm{Xx}^{\mathrm{e}}$ siècle?

Les circonstances politiques ont été décisives et si le legs Aragon-Triolet, initié sous Giscard d'Estaing, s'est traduit, en 1982-1985, non seulement par la création d'un laboratoire du CNRS, mais aussi par l'ouverture de plusieurs postes de chercheurs, c'est grâce à une politique de refinancement exceptionnel de la recherche, comparable à celle qui avait accompagné les «lendemains qui chantent» en 1945, et, deux décennies plus tard, la modernisation des structures scientifiques françaises sous De Gaulle, en 1960-1965. La création de l'ITEM et l'accueil des fonds Aragon-Triolet par le CNRS s'inscrivent, à leur modeste échelle, dans les grands investissements qui ont été déployés, vingt ans après la période gaulliste, sous la présidence de François Mitterrand, en faveur de la recherche et notamment, cette fois, en direction des sciences de l'homme et de la société. C'était la troisième grande vague de soutien de l'État à la recherche publique, dans le cadre d'une périodicité de vingt ans qui était alors perçue, conformément aux recommandations du «Plan», comme le cycle normal de remise à jour des infrastructures scientifiques du pays. Cette troisième vague fut aussi la dernière : le rendez-vous de 2005 n'a pas eu lieu, et on l'attend toujours. Pour éviter à l'avenir toute discussion pénible sur le sujet (ou sur d'autres du même type), l’État y a trouvé une occasion rêvée de faire coup double en matière de restrictions budgétaires : non seulement le refinancement préconisé est passé à la trappe, mais le Commissariat général au Plan (créé en 1946), depuis longtemps dans le viseur de la pensée libérale, est démantelé en 2006. En langage syndical, cela s'appelle «casser le thermomètre pour faire baisser la fièvre».

S'il semble donc bien établi que le lien historique entre le legs Aragon et l'émergence des recherches en génétique littéraire n'a rien eu de fortuit, il reste à s'interroger sur la manière dont l'écrivain, en organisant la transmission de ses archives, a pu construire et communiquer son point de vue personnel sur les études de genèse, l'analyse de ses manuscrits et les processus d'écriture de ses propres œuvres. Comme toujours avec Aragon, la vérité avance masquée, les contradictions ne sont pas rares, l'ironie n'est jamais tout à fait absente des déclarations les plus sincères et, au final, les convictions intimes de l'auteur ne sont pas faciles à préciser. Les démarches de 1975-1977 en vue du legs avaient été précédées, en 1969, par la publication d'une sorte d'avertissement un peu énigmatique : Je n'ai jamais appris à écrire ou les incipit2. Cette autofiction théorique, agrémentée d'une foule de dessins autographes, était parue chez l'éditeur d'art Skira, en Suisse francophone, dans la prestigieuse série dirigée par Gaëtan Picon, «Les sentiers de la création» : une collection dont l'intitulé frappe, rétrospectivement, par le parti pris on ne peut plus «génétique» qui s'y affirme. Qu'Aragon ait choisi d'y publier

2. Louis Aragon, Je n'ai jamais appris à écrire ou les incipit, Genève, Skira, coll. «Les sentiers de la création », 1969.

\section{L'impraticable «mode d'emploi» du legs}




\section{Un événement considérable}

\section{Aragon fait don au C.N.R.S. et de documents relatifs à son}

I Centre matlanal de la recberche scientifique annonce aujourdaci pur potre camarade Louls Aragon vient de lal raire dosation de ses colleetions de manuserits et do epcubsents. ottert en propritite au CNRS, cet en.

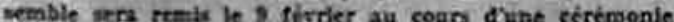
qui se deroulera a won sitse, qual Anatole-France, seion tne dicklan da Centre diaistoire et danalyse des manuscrtts modernes Cent les aetivites regroupent depais 197t, rue te Riebellea a Paris, non loln de la demeure cu Stendhal ectrit ite Roace et le Nols $\rightarrow$. une trentalne d'universitaires de eberchears et de technieiens.

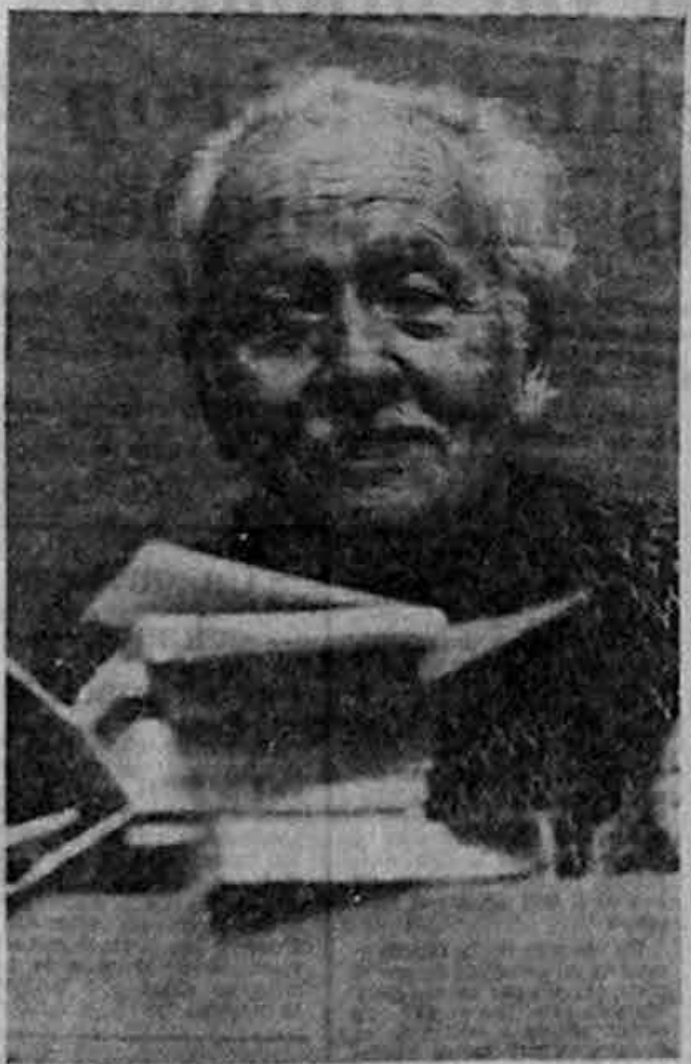

Cis trinerval reves voe porite costidfinbie en rawop curved de ls plisce occuper fir Arscen cand les lettres fruscalests of is eulturs de 5 . the paye dipgle stus go widrant: nos: Une reobide rat: envele du eratid serivata im-

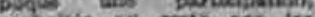
boubalus a rece par ies iclenurigoes, atas traviux ot de clavertines:

cuet une tapen de reconnal. Gidenatication, do datavos travall des chercheurs: des actences trumatines, helins pas toujears appreote a sa funte valeur par ios pouvolis pubilice knns. cette donation permet un elargesement do ia rosation du CNRB, non pius seulement tnititution de zecherche tondamentale mals crition Iittitralre.

Les collections confites au CNRs toptesentent is inuste Con potetes tokettes scs premles covies rumthilsters juricres

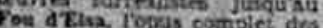
inales en prom, des Cloches de Rise au Thisite Romun : un mintaserit ce nourclies inkatites $y$ rompris $x$ porte. ef des Carnees de travan doot Fedstenas posisat etre, a fuste tires doupconate. A cos de petits towayera un los eire partie de ts cotrespon: cance dsta propileit do in Bibtiotheque boucet.

Pendant naturel do ces prtcevises archivos lawre tzil moles, as tocal vingt-cin manculs de rocanno - le

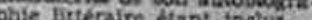
ghie corresire tant inctus ment oele entruteuse aveo Max Jacos, Pavinan conive

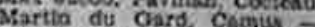
Fexception de ia correspon. dance avec Mainkovist ofter? par Aragon an Pobde Mala. kovald of Moteou - un youma) intime josqu'alons recte incotand In faut inciare dahs cetto somme les manuserits ou dextris dfdies aux deux ferlouins par lecus atmit artis. Len en portscullez Chagait. dre Bretor. Is travall dinventare de fouvre doragon est ootnel a Keorivain Jear Riatst, celai drito Triolet Michel Apel-skuler.

La aimple curlosite, stnon be polvasins interis soofeve par Prexistence a preset connto de cos iatdis autortse of crobte Qu'Ts vant tire un Jour pour partie vabies, on pas ofumet que cecte pubticatio sera rendue pocrible pous re

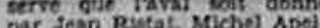

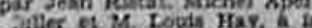
fois dirnctear do recherche is cover et directeur du Centro chictotre it danozice des inandacties moderms.

Reflexion critique - Oa comprend matnitenasat nelles tont avolr acods its chrrcheurs, dont lairc dract ment des Textes sodtrnts consitute cette annete au CNRS, vetend A lexplotation de manukrits lies depuls ia Premure Gecrre mondiale a jinvention pottique, il eat vrai par notro pays

Les recherches textuclles gur colte couvre i double entret. putqureite concerne auss bien Atapon quzisa Trotet, Det: metront ins artloulation plus mionnele, sinon phes ious. ple, des travaux aisperses cuaqua proseni entre aiftesont poue fonction do collec. ter des donntes et de proti. der $\mathrm{A}$ une anatyse documen. taire, de recrubor de facon prevolir los modalited daceis Li reote. II sagit en loc. earrence doun ettort conslde. rebse deploye dans to champ cheorkque ayes bien que lin: - istigue Le compto readu phenominos a maltrlert en
pistoire des idess Texamon hilstolre des idfes, Texamon dee micanime situd a Torit cine du texto declenchiment des prosesura couvent complexes of contra. diotoires guil concourent is is nourrir dans son avancte, odvreat des perspectives do recherche, Andues certes, mais then de motns que pasilon. nantes, cancretes et moblies.

Par allours, un controle incedit de ces exments osser. ves dans leurs interactions con of sens in sagit dexami. ner des temoignaged mate. rels confronte a leurs is rorets asispante ") merito do retenir lattention ivines dos erchives de Jecrivain setraites: en retour, confrontation des résulats aveo Jearvats lus-meme, dont ine ces originaliten do l'ceuvre $\overrightarrow{-}$ letcres mocernes - est illustrte par la profondeur et in conistance de sa teflexion pubituse dans le dotnhine do la

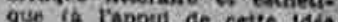

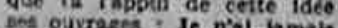
appris pits Jabats men ies ines. nheque de ger cantolet. Chro. motris de con cante of hes to ment is vertithation pratse do in ftrotic des motagye

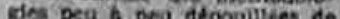
lear mode derporilies de que pournate zits contuste whe un inguter protis dans

Fig. 1 : «Aragon fait don au C.N.R.S.», L'Humanité, 17 janvier 1977. 


\section{les collections de manuscrits}

geuvre et à celle d'Elsa Triolet

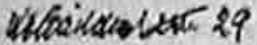

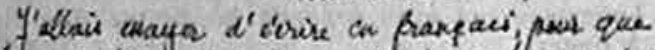

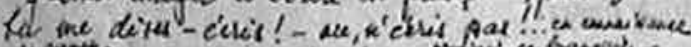

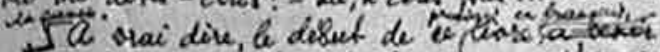

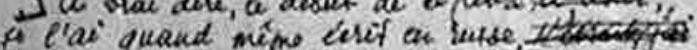
(5)

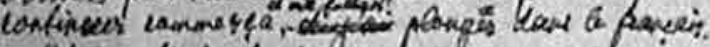

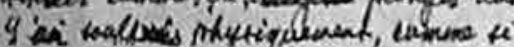

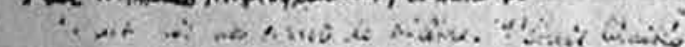

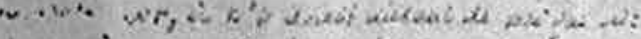

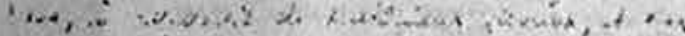

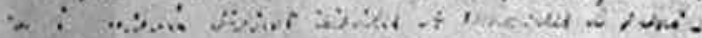

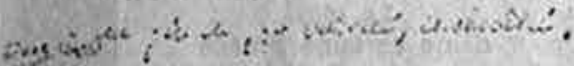

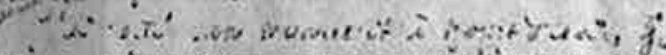

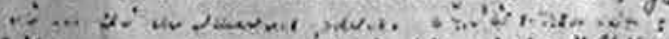

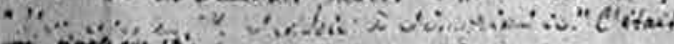
Preface aus Prescict scever in terte eisen. tiet getra Triolet? dremtre en mancelt...

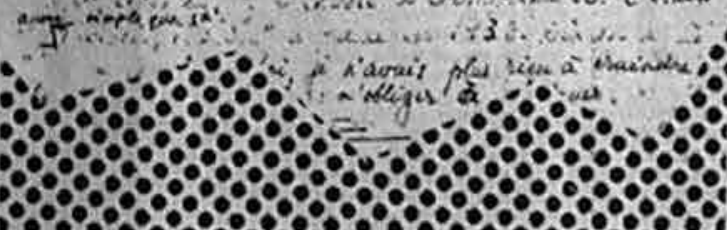

\section{4}

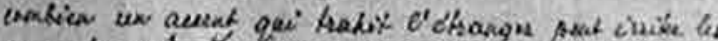

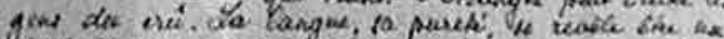

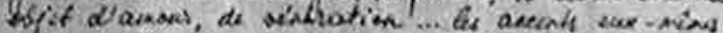

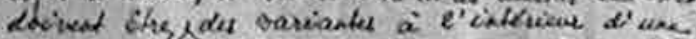

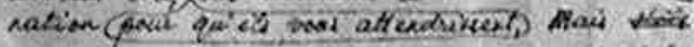

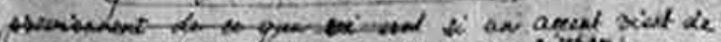

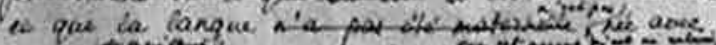

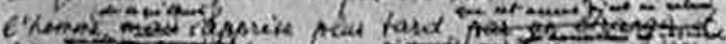

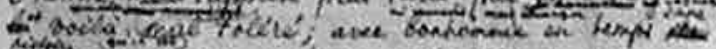

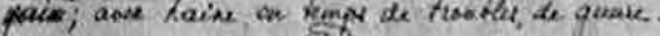

$$
\left.(\mathrm{i} i)^{*}\right)
$$

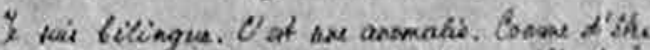

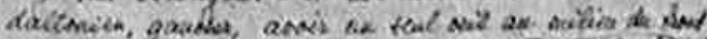

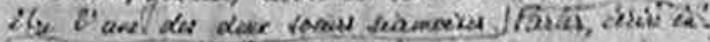

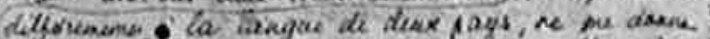

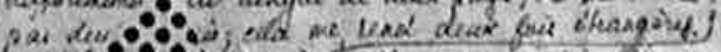

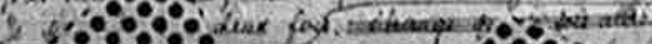

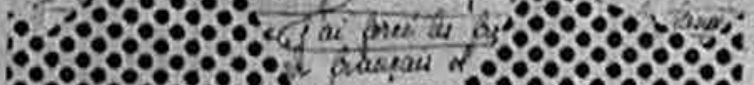

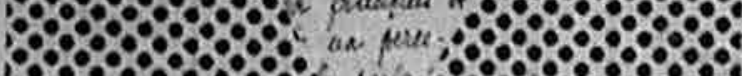

Cavites territoles dos saien. cos bumaines

\section{Offert au pouple} français

If ve trocive gae les recher: ches en cours et ixvealit of ces acienses appoinena radre. wion ou av molns la solldarite. diun vace retesa de colla. boratlons, it mibes is plus lonsue chiance te is pate. wasere manate iu plus pretiritex teisine and

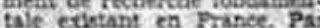
is meme a es offert a peuple friacals. In sinicts of facon lozioue cans jutmeratre exceptioninelement kcond de Terivain et cu mintant if volutionhalize, eul a contri. bod de tacen remurgtabie a. diveloppemieat de la lange francalie

I raut to rettrer peut-tiso

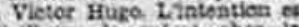
chantear a mese cha is aeur pos des cates

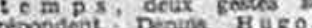
croson repeleste toe

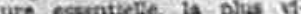
cure de notro notise de no the rom do notre serture

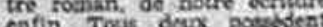

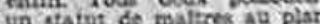
de la creation : lecus cruvres aertictiont toutes do no axpiratiogs nationales Lucien CURZI.

do the ont do $2 \mathrm{a}$ mise sitra (196)

LES " UNE 》 HISTORIQUES de "Itumanité 》 jer cofrket (190s a 1959) 20 COFFRET (1959 a 1947$)$ 30 COFFRET (1947 a 1958)

4. COFFRET (1958 a 1968)

ts comems rovr to verts Ansontoa 1

(100) 200.020

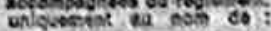
qiapt revatickes

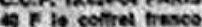

- neacssiz vort celaure e

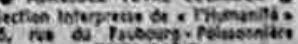
rowon pintis 
une sorte d'art poétique, en forme d'autobiographie littéraire rêvée, n'est évidemment pas indifférent si l'on considère le texte comme une sorte de préambule ou de mode d'emploi qui concerne non seulement l'œuvre mais aussi les archives de travail. Car il s'agit bien d'un métadiscours sur la manière dont l'écriture survient et se développe jusqu'à l'œuvre achevée : au fond, rien de moins qu'une sorte d'approche génétique, avant la lettre.

Mais, d'un autre côté, beaucoup de lecteurs ont remarqué, avec raison, qu'Aragon, loin de nous offrir la bonne méthode pour lire et comprendre ses archives, semble s'ingénier à en compliquer l'élucidation ou même à en rendre impossible l'interprétation. À force de nous expliquer sa mystérieuse méthode - les incipit - et d'en préciser les paradoxales idiosyncrasies, l'écrivain forme autour de ses archives de travail une sorte d'écran de fumée si opaque que les généticiens les plus ardents risquent bien de perdre courage. À sa décharge, on peut dire, bien sûr, qu'Aragon avait de qui tenir. Victor Hugo avait inventé la conservation et la transmission des manuscrits littéraires pour que les chercheurs de l'avenir puissent se ressourcer au jaillissement de son écriture. Mais dans le même temps, il n'avait légué de ses propres archives que la part la moins laborieuse, conservant les débuts fulgurants et les dernières phases démiurgiques, détruisant les notes, les manuscrits de travail et les brouillons intermédiaires : en cherchant délibérément à édifier l'image d'une création géniale et souveraine, une sorte de corpus idéal par défaut qui finit dans bien des cas par rendre l'étude de genèse difficile ou impossible. De même Aragon semble n'avoir voulu, ironiquement, léguer ses dossiers de genèse que pour en interdire l'intelligibilité en nous fournissant un mode d'emploi piégé. De deux choses l'une : ou il dit vrai ou il ment. Si nous croyons à ce que nous révèle le métadiscours des Incipit, il devient inutile de plonger dans les archives : le travail est fait, l'essentiel est dit, et nous n'avons rien de plus à découvrir ou plutôt à vérifier dans les manuscrits que ce que l'écrivain nous dit lui-même des processus de création qui ont présidé à son œuvre. Ou bien nous n'y croyons pas, et nous devons alors nous demander par quelle autre méthode interpréter les archives, en commençant par chercher à comprendre pourquoi diable l'écrivain s'est ingénié à nous fourvoyer. À moins bien sûr que ladite contradiction n'en soit pas une, que le mentir soit vrai et que le piège soit l'issue de secours. Ce serait le cas si Les Incipit étaient à comprendre comme une œuvre de fiction, ou du moins comme un texte où l'intelligibilité revendiquerait le droit à se prévaloir des moyens de la fiction.

\section{L'auteur, l'analyste et l'écrivain}

Que nous dit Aragon dans Je n'ai jamais appris à écrire? Il nous explique que l'écrivain se met à écrire sous la pression d'une phrase lue, d'une formule entendue, ou d'une phrase reçue en rêve qui va jouer le rôle de matrice, de germe, de déclencheur ou de générateur primaire de la rédaction. L'incipit est ce «don» qui initialise l'écriture, ce cadeau des dieux, semblable au «vers donné» dont Paul Valéry parlait dans les mêmes termes : il ne relève jamais d'un calcul de l'esprit, mais s'impose comme l'impact d'un choc aveugle sur la sensibilité. Comme pour Valéry, il est de nature dionysiaque. L'incipit est le point de départ de la création, l'écrivain au travail ayant ensuite à laisser se développer, autour de ce point, en toute lucidité et moyennant un contrôle de chaque instant, les contenus d'une œuvre qui devra être constituée, en chacune de ses composantes, d'une matière et d'une forme aussi pures que celles du don initial. Ce choc primal initialise l'écriture sans que l'incipit originaire coïncide nécessairement, ni généralement, avec ce que seront les premières lignes du livre. 
Car, une fois l'énergie rédactionnelle amorcée par l'impulsion de ce don, l'œuvre s'écrit de toutes parts, en amont aussi bien qu'en aval de ce début, comme si la totalité finale du texte préexistait hors du temps, avec l'évidence vertigineuse d'une sorte de c'était écrit :

Mes romans, à partir de la première phrase, du geste d'échangeur qu'elle a comme par hasard, j'ai toujours été devant eux dans l'état d'innocence d'un lecteur. Tout s'est toujours passé comme si j'ouvrais sans en rien savoir le livre d'un autre, le parcourant comme tout lecteur, et n'ayant à ma disposition pour le connaître autre méthode que sa lecture. Comprenez-moi bien, ce n'est pas manière de dire, métaphore ou comparaison, je n'ai jamais écrit mes romans, je les ai lus 3 .

Rédiger, pour l'écrivain, revient à lire un livre qu'il s'agit de recopier sans se tromper, aussi fidèlement que possible, en se laissant guider dans cette lecture-écriture «non par un raisonnement, mais par une rencontre de mots, ou de sons, la nécessité d'une allitération, une logique de l'illogisme, la légitimation après coup d'un heurt des mots. L'accident expliqué ${ }^{4}$ ». Dans cette tâche, l'auteur au travail est formé de deux instances : d'un côté, il y a l'écrivain qui a reçu l'impulsion dionysiaque et qui, sous son ascendant, rédige en se bornant à lire le texte de l'œuvre, comme s'il savait déjà par cœur, le chemin à suivre, les yeux fermés, pour le parcourir jusqu'à son terme; et d'un autre côté, l'analyste (l'instance apollinienne) qui tient, à chaque instant, l'automatisme de cette réminiscence sous haute surveillance pour éviter au rêveur éveillé la menace d'un faux pas, le risque de perdre sa route et de s'égarer.

Mais, moyennant l'équilibre de ces deux instances (évidemment empruntées à Valéry qui les tenait lui-même de Nietzsche), c'est bien d'une écriture-lecture de nature quasi automatique, sans plan ni véritable brouillon, pratiquée au fil de la plume, avec une énergie toute stendhalienne, qu'Aragon nous parle comme du mode de rédaction qu'il a suivi pour toutes ses œuvres. Pour le démontrer, il développe dans Les Incipit un véritable panorama rétrospectif : de Quelle âme divine! (1903) à son dernier roman publié, La Mise à mort (1965), de la saga du Monde réel à la mythique aporie de La Défense de l'infini, il retrace, étape par étape, l'itinéraire intégral de son parcours créatif en le présentant, malgré toute sa diversité, comme le triomphe réitéré et finalement uniforme du même scénario : la mystérieuse opérativité de l'incipit. Sauf exception (Les Beaux Quartiers) conçue comme telle dans le seul but de confirmer la règle, la conformité au modèle de l'incipit constitue la norme.

Une sorte d'anomalie fonctionnelle se cache pourtant au cœur de cette régularité, car celui Le passager clandestin qui n'a jamais écrit que sous l'influence de l'incipit, en laissant s'opérer le miracle du don initiateur et de l'œuvre déjà écrite, renvoie à un «je» qui n'est pas Aragon. Si l'auteur qui va finir pas endosser socialement la responsabilité du texte publié se compose d'un analyste apollinien et d'un écrivain dionysiaque, aucune de ces trois instances virtuelles ne coïncide avec celui qui peut être considéré comme l'authentique concepteur de l'œuvre. Le véritable créateur est ontologiquement ailleurs : c'est un autre radical, fantomatique et révolu, qui possède les contours fugaces d'une silhouette absente à laquelle l'auteur fictif d'aujourd'hui a cherché, il y a très longtemps, dans sa jeunesse, à s'identifier. Chaque cadeau initiateur d'une 
nouvelle œuvre n'est que la répétition ou la résurgence épisodique du cadeau des cadeaux. Au sens strictement privé qu' Aragon donne à cette procédure d'initialisation, écrire consiste à célébrer un retour à l'incipit des incipit, c'est-à-dire à la présence spectrale et native de Maldoror : altérité irréductible et figure paternelle qui incarne le principe anhypothétique et l'analogon formel de toute entrée en écriture, l'Incipit-Maldoror fait de l'acte d'écrire un sursaut contre l'effroi de n'être rien. Commencer à écrire, laisser s'accomplir le miracle immanent de l'Incipit repose sur un potentiel énergétique latent : ce que le désaveu rimbaldien de soi-même ( «je est un autre») assure comme énergie à celui qui ne tient sa faculté d'écrire que de l'expérience d'avoir aboli son identité pour être le (fils du) comte de Lautréamont. Déni sadien et surréaliste autant que rimbaldien, cette récusation du moi (et ses accessoires : la perte du nom, la quête éperdue du père, l'illégitimité, etc.) n'ont rien ici d'un trait d'esprit ou d'une coquetterie : c'est un destin. Pour toutes sortes de raisons intimes, aussi impérieuses que douloureuses, et qui tiennent à son roman familial, le trouble de l'identité et l'angoisse de la filiation chez Aragon sont le contraire d'une posture. En revanche, ils ne se soldent pas par le contraire de l'imposture : l'authenticité du trouble appelle à un renversement du véridique, à une passion pour le mensonge, à un pari pour le travestissement, à un salut qui ne se trouve que dans l'artifice et l'esquive, à une fuite sans retour dans l'univers du faux-semblant. L'incipit renvoie à l'épreuve initiatique d'une dépersonnalisation et d'un dédoublement où se reconstitue le lien fondateur d'Aragon avec ses enfances rêvées, l'imaginaire de son propre commencement, l'idée folle d'un engagement absolu dans l'écriture : la force désespérée de tout miser sur le semblant, le courage de s'embarquer par effraction, sans billet, en devenant le passager clandestin de sa propre existence.

Cette puissance de la duplicité est si constitutive de l'être-écrivain que la valeur injonctive et performative de l'incipit se combine dès l'origine avec l'affirmation d'un droit imprescriptible à la contradiction («je n'écris jamais que pour me contredire»), ce qui conduit Aragon à défendre avec autant de vigueur l'hypothèse selon laquelle le principe de l'incipit peut tout aussi bien être supplanté, le cas échéant, par une «préconception» du roman, comme le montre la genèse des Beaux Quartiers, récit méticuleusement agencé selon un plan, précisément dans l'intention de «contredire » ceux qui avaient critiqué l'absence déplorable de construction dans Les Cloches de Bâle. Au total, le lecteur médusé des Incipit est sommé de se laisser convaincre, ou plutôt de se laisser subjuguer, par une méthode (la négation du principe de non-contradiction) dont vous comprenez à demi-mot qu'elle a pour but de vous faire perdre définitivement votre chemin, et même toute idée de chemin, de faire de vous un égaré :

Chacun de mes livres contredisant le précédent, le parti pris du précédent, mais rétablissant ainsi la continuité de tous. J'écris cette note pour tenter d'introduire dans l'esprit du lecteur une méthode qu'il appliquerait par la suite sans que je l'y sollicite du Paysan de Paris au Fou d'Elsa, et au-delà. Jusqu'à ce roman-ci ${ }^{5}$.

Prolégomènes à toute lecture des manuscrits qu'il nous transmet sept ans plus tard, Je n'ai jamais appris à écrire porte donc le principe du «mentir vrai» à son point d'incandescence le plus destructeur, avec la malice d'un incendiaire qui mettrait le feu à sa propre maison : nous devons comprendre que les archives sont en flamme, que le brasier les consume depuis 
toujours et que, pour les déchiffrer, le lecteur devra se débrouiller avec un tas de braises et de cendres. À prendre au sérieux, et en détail, ce que Les Incipit nous disent des «sentiers de la création », nous voilà donc placé devant un pur déni : il nous faut admettre que toute étude de genèse se trouve a priori révoquée en doute avant même de pouvoir être entreprise, comme si Aragon cherchait à formuler par anticipation un démenti formel au bien-fondé du geste de transmission qu'il se prépare à annoncer : je vais vous donner tous mes manuscrits, mais ne cherchez pas à les comprendre, ce serait peine perdue, vous ne pourrez jamais rien en faire... À moins justement qu'il ne s'agisse de tout autre chose dans cet avertissement : que la volte-face ne porte pas sur la faisabilité de l'entreprise ni sur sa pertinence, mais sur ce qu'Aragon pressent comme un malentendu central quant aux enjeux. Il se pourrait bien que la leçon déroutante des Incipit ait quelque chose à voir avec ce qu'Aragon n'écrira pas mais dira, huit ans plus tard, dans le fameux discours de 1977 par lequel il présente solennellement son legs au directeur du CNRS.

La transcription de ce discours, publiée en préface aux Essais de critique génétique 6 , n'est pas facile à synthétiser : fidèle aux paroles improvisées par l'écrivain, c'est un texte émouvant et même passionnant, embarrassé de digressions et d'idées intempestives, dont on peine souvent à suivre la ligne argumentative. Mais c'est aussi un texte résolument programmatique qui démontre avec force qu'Aragon, en léguant ses manuscrits et ses documents de travail, sait parfaitement à quel type de recherche ils sont destinés : une étude de genèse, une investigation sur les processus les plus secrets de l'écriture. Et Aragon précise qu'un tel projet coïncide justement avec ce qu'il désire le plus intensément pour ses archives : c'est le destin qu'il veut pour elles. L'approche génétique est décrite avec précision, dans ses réquisits spécifiques, comme une méthode exigeant une véritable plongée dans l'avant-texte de l'œuvre. Il s'agit, dit-il, de

[...] pouvoir mettre sous les yeux des lecteurs passionnés que sont les vrais chercheurs, non pas le texte en état de publication, toute retouche faite, et mené par son auteur à l'état de livre, c'est-à-dire de marchandise livrée, mais, surprises pendant son écriture, les hésitations de l'auteur, ses secrètes démarches, ses erreurs même... voire ses brouillards, encore un air de brouillon, par-ci, par-là...7

Et de surenchérir : les brouillons, oui bien sûr, mais pas seulement les brouillons; beaucoup d'autres pièces doivent être rendues accessibles aux chercheurs pour qu'ils puissent précisément penser les brouillons et les interpréter dans toute la complexité des processus d'écriture qui ne relèvent jamais de logiques simplement techniques. Chaque rature est une aventure émotionnelle et esthétique qui met en jeu des dizaines de variables dont le secret se trouve caché dans d'autres archives. C'est cette totalité qu'il s'agit donc de réunir et de léguer, pour, dit-il,

6. Louis Aragon, «D’un grand art nouveau : la recherche», Essais de critique génétique, Paris, Flammarion, 1979, p. $7-19$.

7. Ibid., p. 9 . 
mettre à la disposition des chercheurs d'aujourd'hui, non pas seulement un certain nombre de manuscrits, au sens des éditeurs, mais les papiers de ma vie mentale, les dossiers complexes sans lesquels, à mon sens, ce qu'on appelle le manuscrit n'est que bien peu de choses... ${ }^{8}$

Beaucoup d'autres passages de cette intervention orale d'Aragon mériteraient d'être cités et analysés de près : on y trouverait, y compris aujourd'hui, des idées fertiles pour l'avenir de nos travaux. Mais le message essentiel de ce discours tient peut-être surtout dans l'intitulation que l'écrivain a voulu lui donner : «D'un grand art nouveau : la recherche», un énoncé troublant et inattendu dont Marie Odile Germain dit avec raison qu'il s'affirme et s'affiche comme un titre flamboyant qui élève «la recherche au rang des beaux-arts ». Qu'est-ce qu'Aragon voulait dire? L'ITEM et les chercheurs en génétique ont ressenti la formule comme une marque précieuse d'adhésion à leur discipline et comme une valorisation de leurs travaux, mais sans vraiment s'interroger sur ce que l'expression «D'un grand art nouveau : la recherche» pouvait contenir de proposition d'avenir pour repenser la place et l'identité de ce nouveau domaine de compétence. J'ai toujours pensé, pour ma part, que cette formule ne prenait son sens que rapportée à une véritable réflexion sur les finalités de l'investigation en sciences de l'homme et de la société, par opposition à ce que la recherche peut signifier dans les sciences dures et appliquées. En qualifiant de «grand art» le nouvel espace de recherche ouvert par la génétique littéraire, ce que cet intitulé nous invite à définir et à défendre, c'est un nouveau type de légitimité scientifique : le bien-fondé d'une pensée critique créatrice qui s'exercerait de plein droit en tant que pratique théorique à vocation artistique, c'est-à-dire dans le cadre d'un discours qui, pour élucider scientifiquement son objet, aurait résolument la charge de problématiser la nature de ses relations au concept de vérité.

\section{Genèse et critique insciente}

À l'exercice purement logique et rationnel d'une recherche scientifique à vocation démonstrative, Aragon oppose l'hypothèse d'une recherche empathique qui serait capable de simuler la complexité de son objet pour le comprendre de l'intérieur et en renouveler les significations : ce que Flaubert appelait de ses vœux en parlant de «critique insciente». N'oublions pas qu'Aragon s'adresse ici directement à «M. le directeur général du CNRS » dans un contexte intellectuel qui est alors celui du «moment théorique» : une période d'intense effervescence spéculative où l'écriture critique se trouve revalorisée en termes de pratique créative, comme c'est le cas pour toutes les stars de la french theory : Lacan, Deleuze, Derrida, Foucault, Genette, etc. et, à sa manière, Roland Barthes qui avait d'ailleurs été chercheur au CNRS avant de passer à l'École pratique des hautes études, puis au Collège de France. Une époque où, réciproquement, les créateurs et les romanciers se font aussi théoriciens, comme Georges Perec, lui-même ingénieur au CNRS... Aragon ne s'y réfère ni directement ni indirectement, mais son exorde revient à plusieurs reprises sur les «jeunes écrivains » contemporains dont le talent artistique constitue selon lui un gage d'excellence dans le domaine de l'analyse critique et génétique, comme si l'étude des manuscrits, l'élucidation des processus d'écriture et le récit de genèse relevaient indissociablement de qualités hypothético-déductives qui sont celles de la science et de qualités oniriques et fictionnelles qui sont celles de l'art. Le «grand art» qu'il

8. Ibid., p. 14. 
invoque ne relève pas du discours de vérité mais de son épochè, de sa révocation en doute au profit d'une nouvelle intimité épistémologique avec les grands maîtres de l'illusion. Parler de «grand art» ne consiste pas, comme certains ont pu être tentés de le faire, à revendiquer pour la recherche un statut artistique qui ferait du chercheur ou du critique, donateur souverain du sens, un concurrent du créateur. Cela consiste au contraire à installer au cœur de la démarche d'investigation la puissance de problématisation qui est celle de l'incipit : entrer dans la connaissance de la genèse de l'œuvre par la même procédure d'anéantissement du «je » que celle par laquelle l'écrivain peut entrer en écriture.

Il n'est pas certain que la critique génétique ait su tirer le meilleur parti possible de ce présupposé pour apprendre à déployer la part la plus créative de ses compétences. En revanche, quant au traitement scientifique du fonds Aragon lui-même, l'approche génétique a su donner la preuve qu'elle était à la hauteur de sa mission. Dans des actions coordonnées par Jean Ristat et Michel Apel-Muller, avec la précieuse contribution de Renate Lance et de Danièle Maïsetti, les chercheurs de la première équipe Aragon de l'ITEM ont accompli en une vingtaine d'années, jusqu'à la fin des années 1990, un immense travail d'inventaire et d'analyse. Mais une fois ce grand chantier terminé, l'équipe, un peu à bout de souffle, a fini, hélas, par s'auto-dissoudre faute de moyens financiers suffisants pour envisager de passer à la nouvelle phase de recherche qui paraissait s'imposer : déterminer un axe thématique et sélectionner une partie des fonds pour procéder à l'exploitation scientifique des archives qui venaient d'être inventoriées en affinant le classement génétique des documents. Le pôle de recherche Aragon est ainsi resté absent de l'ITEM pendant près de dix ans. En flagrante contradiction avec les engagements pris et avec l'histoire même de notre structure, cette situation paraissait intenable. C'est la raison pour laquelle, en reprenant la direction du laboratoire en 2006, j' ai décidé qu'une des priorités les plus urgentes était de refonder une équipe Aragon dynamique, capable de rassembler une nouvelle génération de chercheurs. En 2008, c'était chose faite : Luc Vigier acceptait d'en prendre la responsabilité et relançait un programme de recherche de premier plan sur cet immense fonds auquel l'ITEM doit tout simplement la chance d'exister.

Artiste, écrivain et chercheur, PIERRE-MARC DE BIASI a dirigé l'ITEM de 2006 à 2013. Il a publié de nombreux ouvrages et articles sur Flaubert, la génétique, le papier, le lexique contemporain, l'histoire de l'art, la médiologie, la révolution numérique. Dernier ouvrage paru : Le Troisième Cerveau, 2018. Directeur de collections chez CNRS Éditions et EAC, rédacteur au Magazine littéraire, il a réalisé plusieurs films pour Arte et plus de quatre cents émissions pour France Culture. Son œuvre plastique a donné lieu à une cinquantaine d'expositions et à sept commandes publiques en France et dans le monde. 